\title{
MAGNETIC PULSE WELDING: LESSONS TO BE LEARNED FROM EXPLOSIVE WELDING
}

\author{
J. Verstraete ${ }^{1}$, W. De Waele ${ }^{1}$ and K. Faes ${ }^{2}$ \\ ${ }^{1}$ Ghent University, Laboratory Soete, Belgium \\ ${ }^{2}$ Belgian Welding Institute, Belgium
}

\begin{abstract}
Almost 50 years after magnetic pulse welding was invented, it is finally finding its way to the private sector, in particular the transportation and refrigeration industries. To support this evolution, more fundamental and applied knowledge on magnetic pulse welding has to be established. Learning from the explosive welding process which is very similar and already thoroughly studied and documented, is one way of achieving this. This paper first discusses why both processes are alike, but not the same. A closer look at the process parameters and especially those of explosive welding learned, that an increasing flyer plate velocity results in a transformation of the bonding interface from smooth to wavy and an increase in hardness at the surfaces of both metals. Welding windows developed for explosive welding are discussed. The reasons for the limitations set to impact angle and collision velocity in a welding window are briefly reviewed. This information can give a hand in the optimization of the parameter settings to achieve sound welds with the magnetic pulse process. To check if a high quality weld is made, several testing methods for both processes are discussed and compared.
\end{abstract}

Keywords magnetic pulse welding, explosive welding, welding parameters, welding window, quality testing.

\section{INTRODUCTION}

This paper is part of an ongoing research on magnetic pulse welding and forming of the Belgian Welding Institute in partnership with CEWAC (Centre d'Etude Wallon de l'Assemblage et du Contrôle des Matériaux). This research aims to demonstrate the industrial advantages of this technology.

Magnetic pulse welding (MPW) is a solid state welding process that uses the power of a high energy magnetic field to create a bond. In the case of tubular products, the magnetic field causes the outer workpiece, also called flyer tube, to collapse on to the inner workpiece (Figure 1a). When this collisiontakes place at very high speed, the high pressure impact results in metallic bonding wherein atoms of the two materials are brought into direct contact.
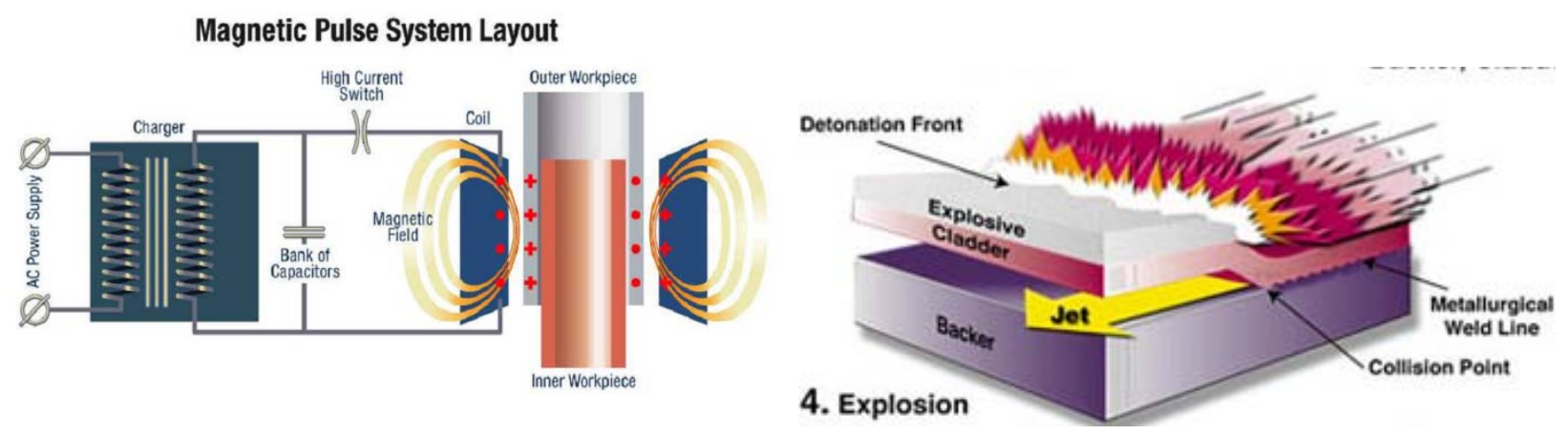

Figure 1: (a) MPW process overview [1] (b) EXW process overview [2]

Explosive welding (EXW) is a solid state welding process as well, but makes use of controlled detonations to force two metals in a high velocity impact, creating a metallic bond (Figure 1b). The bonding principle is thus the same as in magnetic pulse welding although the practical implementation and thereby its applications are different. The explosive welding process was discovered in the late 1950's but was rapidly industrialized worldwide. During the more than 50 years of industrial applications, the process has been continuously improved. This contrary to the MPW process which was discovered a little later but didn't directly find its way to the industry. Therefore it is far less investigated although it has a high potential and some major advantages. MPW is one of the rare processes capable of joining dissimilar metals in a high 
volume product environment. Up to now, any joints between round parts such as tube-to-tube joint and tube-to-rod joint are ideal candidates for MPW. MPW can also be applied to non cylindrical workpieces like flat sheets[3]. But this is outside the scope of our research activities. Because the bonding principles are the same for EXW and MPW a good understanding of the MPW process can be derived from an already existing insight on EXW.

\section{COMPARISON OF MPW AND EXW}

It is generally agreed that the joining mechanism for MPW is the same as for EXW. This cannot only be concluded from the obvious fact that both processes use a high velocity impact to achieve a metallurgic bond. Looking at microscopic images (Figure 2) reveals the appearance of a similar wavy bonding interface as well. In both processes the formation of a jet takes place at the collision zone. The formation of the jet can be assigned to hydrodynamic material behaviour in the bonding zone. The pressure near the point of collision is so high that the material there behaves like a fluid but incompressible and inviscid. The collision of the metals creates a jet consisting of a mixture of metal ions, air and oxides, ejected from the surfaces of both metals[4]. For explosive welding experiments have pointed out that this jet cleans the surfaces sufficiently before welding. Specific surface preparation is therefore not needed. Experiments with MPW however, proved that degreasing the workpieces directly before welding has positive effect in achieving sound welds.[5]
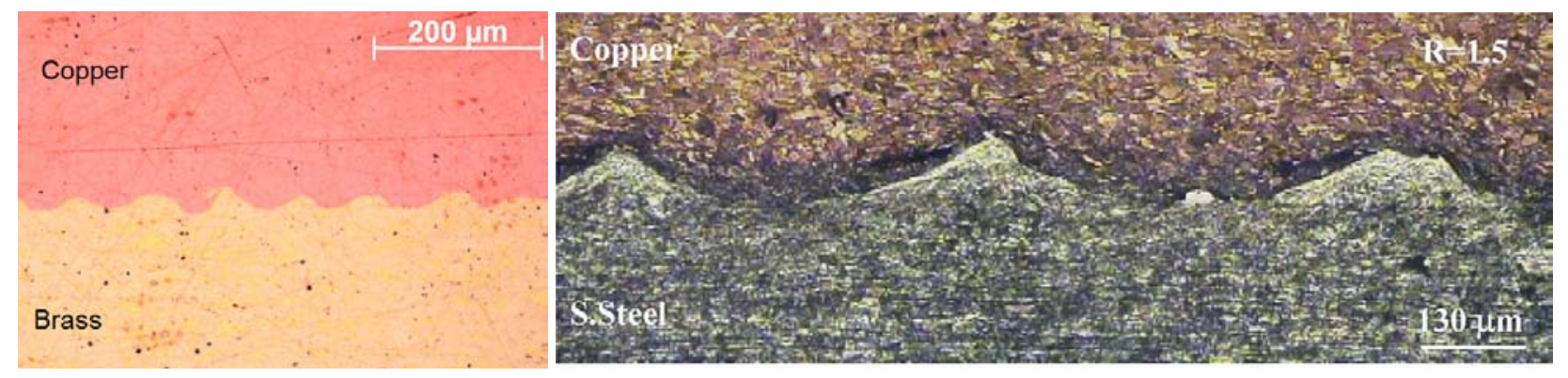

Figure 2: (a) Waviness observed in microscopic images of a MPW interface [6]

(b) and of an EXW interface [7].

The big difference between MPW and EXW lays in the origin of the pressure used to accelerate the flyer plate. For magnetic pulse welding, this is a magnetic field generated by a coil and focused by a field shaper. This field is limited to a small area, of very short duration, clean and safe. The only provision needed to generate it is an electrical current, which is provided by a capacitor bank connected to the electricity net. The capacitor bank charges to a voltage of around 15 to $20 \mathrm{kV}$, and discharges a current of 150 up to $500 \mathrm{kA}$ with a frequency of roughly $14 \mathrm{kHz}$ [6]. The magnetic field will only generate enough pressure under the condition that the flyer tube is a good electrical conductor. There is a limitation in the size of the coil. This is mainly economical, because a bigger coil needs more energy, enlarging all the equipment. Because MPW is so fast (a weld is produced in less than 100 microseconds [4]), clean and easy (once parameters are defined) it is very suitable for automation.

The explosive welding process makes use of explosives to generate a pressure wave, accelerating the flyer plate. Explosives are cheap, high in energy and there is no real size limitation. The downsides of explosives are that they are dangerous, only trained personnel can handle them, most produce toxic gases and the explosion has to take place in the outside or in a specially designed room that can withstand the pressure. The process can weld cylindrical parts with special setups but the easiest and most used configuration welds flat plates.

This frankly means that MPW is limited to weld large series of thin walled, tubular parts that have a high electrical conductivity and EXW is most convenient to weld thick and large metal plates.

\section{WELDING PARAMETERS}

\subsection{Overview}

The welding parameters for MPW and EXW are mostly identical except for those concerning the magnetic field respectively the explosives. In the following table an overview is given of the parameters applied to both processes. Figure 3 shows a schematic overview of the basic parameters. 


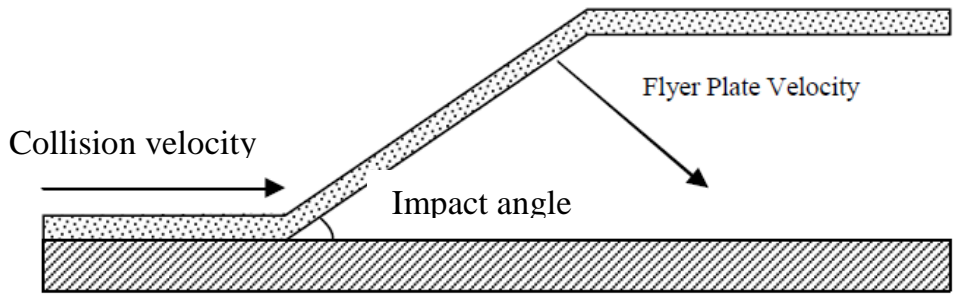

Figure 3: Welding parameters common to MPW and EXW [8]

Impact angle $(\alpha)$

Collision velocity (Vc)

Flyer velocity (Vf)

Standoff

Loading ratio

Anvil

This is the angle formed by the base plate and the flyer plate on impact. For EXW the impact angle is considered constant whereas for MPW the angle starts small and ends big for a parallel setup of the tubes[6]. The impact angle normally is somewhere between $3^{\circ}$ and $30^{\circ}$ [9].

This is the speed of the collision point parallel to the base plate. For explosive welding, it is controlled by the detonation velocity, the speed in which the explosive front travels across the layer of explosives. This parameter depends on the type of explosive used. For both processes the collision velocity is in the order of 1500 to $3000 \mathrm{~m} / \mathrm{s}$ [4]. The collision velocity should remain lower than the sonic velocity of both flyer and base plate.

This is the speed of the flyer plate. There is a proportional correlation between impact angle, collision and flyer velocity $\mathrm{Vf}=\mathrm{V} \mathrm{c}^{\star} \sin (\alpha)$ [9]. For both processes this speed is around 250 to $500 \mathrm{~m} / \mathrm{s}$ [4].

The standoff distance is the distance between flyer plate and base plate (EXW) or between flyer tube and core (MPW). This standoff distance is generally expressed as a multitude of the flyer plate thickness. It is normally between 0,5 and 3 times the thickness [10]. Because explosion welding can weld thicker plates, the average standoff distance is larger as well.

Specific explosive welding parameters:

This is the type of underground the base plate is lying on. The anvil has to make sure the base plate does not move downwards during the explosion. For MPW this translates in the yield strength, stiffness and hardness of the inner part. It can be a solid core or a tube with a mandrel.

Explosive mass to flyer mass ratio. The higher this ratio, the more explosives used. And thus the more powerful the explosion will be. For MPW this can be compared to the charging voltage and energy level of the machine because these parameters will also influence the force of the magnetic field.

\subsection{Influence of process parameters for explosive welding}

In several papers ([10],[11],[12]) it is reported that increasing the flyer plate velocity on impact transforms the bonding interface from smooth to wavy. This can be realized in two ways. Increasing the loading ratio increases the pressure the explosion exerts on the flyer plate, thus resulting in a higher acceleration. Increasing the standoff distance gives the flyer plate more time, thus reaching a higher end speed. Note that increasing the standoff distance to much can make the flyer plate decelerate again. Low speeds do not result in a wavy surface $(<200 \mathrm{~m} / \mathrm{s})$. Once waves appear, further increasing the loading rate or the standoff distance increases wave length and amplitude. With increasing flyer plate velocity the following interface types will occur: straight, smooth, small waves, larger waves, waves with vortices, waves with solidified melt pockets and continuous fused layers. The most desirable interface is the wavy or transition bond without any apparent intermetallic layers. It is well known that an intermetallic layer will most probably lower the ductility and cause brittleness [11]. The benefits of the wave formation are: a higher contact surface, higher mobility of atoms and dislocations and effective removal of contaminations. Some research [12, 13] points out however, that the waviness of the interface does not have an effect on shear strength. Sound welds were made without waves in the interface zone as well.

For EXW al three parameters $(\mathrm{Vc}, \mathrm{Vf}, \alpha)$ are considered constants. This is not the case for MPW as can be concluded from microscopic imagesof the entire length of the weld (Figure 4). A continuous change in waveform (amplitude and period) can be observed and is attributed to variations in flyer velocity and/or impact angle. A detailed analysis of the wave geometry could tell something about the local flyer velocity and impact angle. 


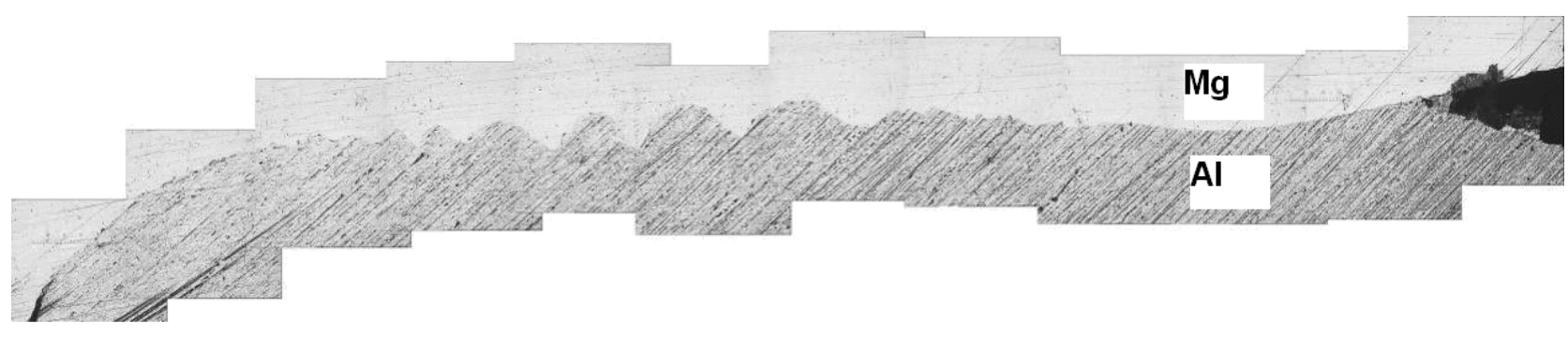

Figure 4: Microscopic image of a MPW over the entire length of the weld [4]

Another effect of increasing the standoff distance is the increase of hardness in the interface layer. Due to plastic deformation, there is always an increase of hardness along the interface layer. The higher the plastic deformation, the higher the increase in hardness (Figure 5a). The measurements also show an increase in hardness on the outer surfaces of the plates. There is a compression here as well due to action - reaction. Analysis of the microstructure of interface layers shows that the grains are elongated in the explosive welding direction (Figure 5b). Heat treatment of the weld zone can recrystallize these grains resulting in a higher shear and bending strength. So far there has been no report of this phenomenon in MPW.
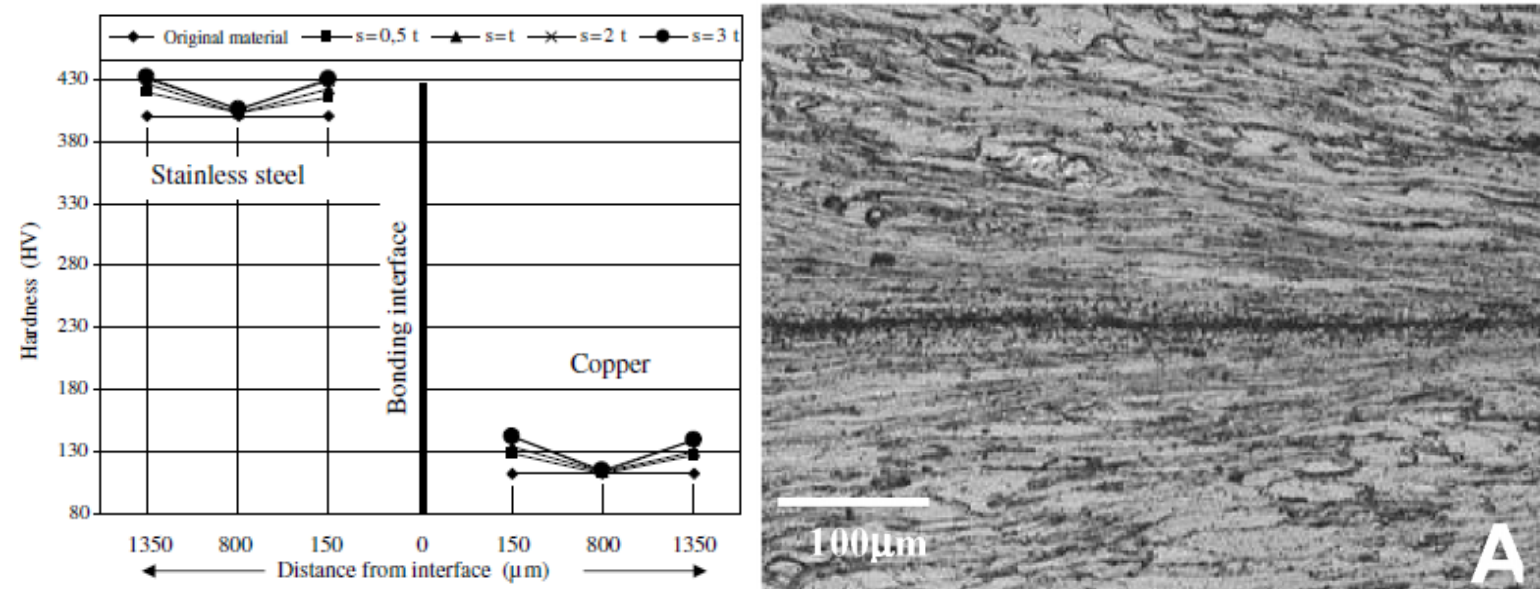

Figure 5 (a) Hardness distribution for a metal couple welded at different standoff distances [10].

(b) microstructure of elongated grains [12]

Besides the loading ratio and the standoff distance some articles also discuss the importance of the anvil. Different anvils are used: sand and steel plates varying in thickness and hardness. The conclusion is that the softer the anvil, the higher the loading ratio needed to establish a sound weld. No welds could be formed for an anvil of sand nor for thin plates[11, 12]. Translated to MPW it is very important that the core material has a hard yield strength and is as stiff and hard as possible, so no bonding energy will be lost in plastic deformation of the core or inner tube. This conclusion was also drawn directly out of several MPW tests reported in [5].

\section{WELDING WINDOWS}

Welding only takes place if the process parameters or within an optimal range. As stated before, an optimal weld has a wavy or transition bond without intermediate layer. To achieve this, the collision velocity has to be slightly greater than the transition velocity (velocity at which waves start to form) and less than the sonic velocity of the work pieces. The collision velocity has to impart the kinetic energy and fluid behaviour for jet formation and should result in a collision angle corresponding to small wavy interface. Too high velocity could cause interface melting. For explosive welding, these parameters have been studied and resulted in several welding windows such as Figure 6. 


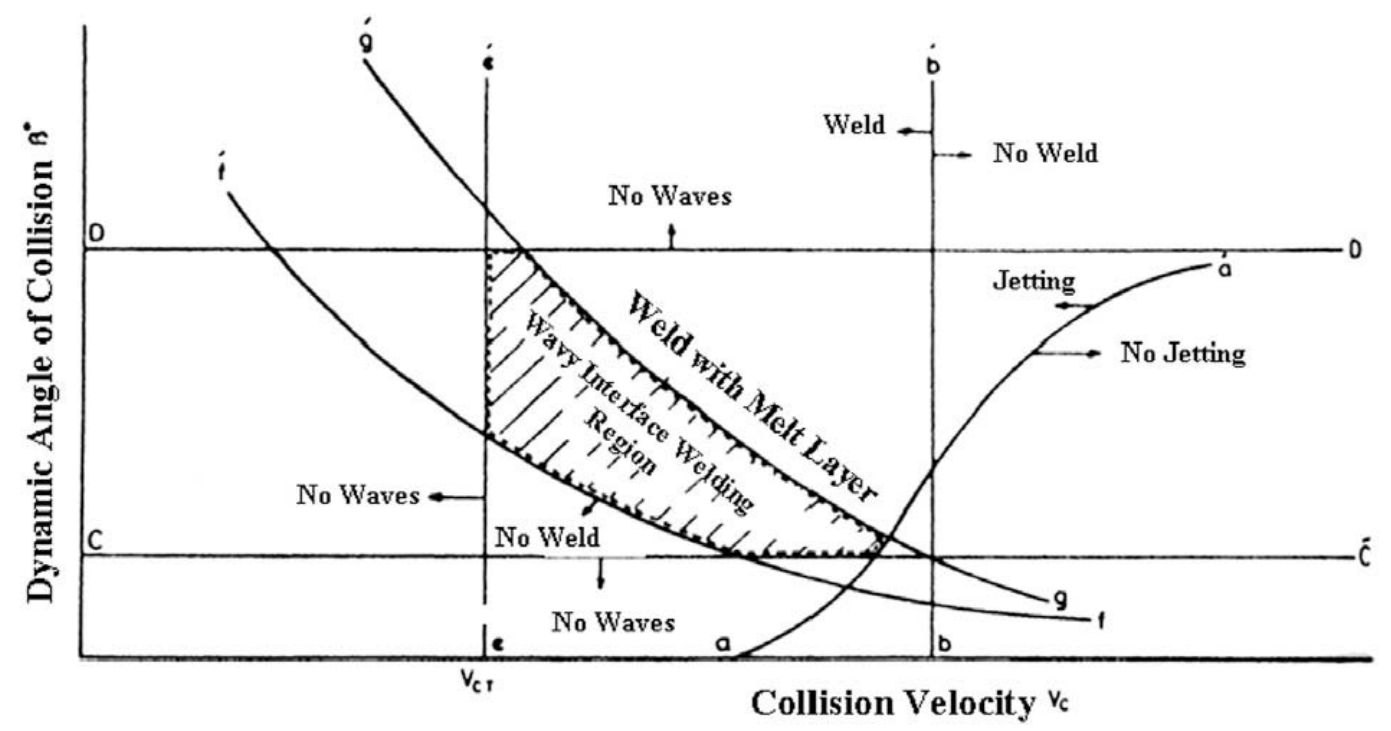

Figure 6: Generic welding window[9]

The welding windows normally plot the impact angle in the ordinate and the collision velocity in the abscissa. One of the most important conditions for welding is the formation of a jet. Jetting has to occur at the collision point to achieve welding. In theory if the velocity of the collision point remains subsonic jetting will occur. In practice however, a minimum angle is required to satisfy the pressure requirement. That is, the pressure must be sufficient to exceed the dynamic elastic limit of the metal to ensure deformation of the metal's surface into jet. In Figure 6 line aa represents the critical impact angle which is necessary for jetting. Line bb represents the upper limit of the collision velocity depending on the sonic velocity in the materials. The lower and upper limits of the impact angle were experimentally determined at around $3^{\circ}$ and $30^{\circ}$ respectively for a parallel geometry. The line ff counts as the lower limit for welding. Above line gg, interfacial melting will occur. All these lines are material depended and have to be calculated for the specific applications. As can clearly be seen from Figure 6, approaching the upper limit of the collision velocity restricts the choice of other parameters within the welding window [9].

These welding windows are material and geometry dependent and can be used to make a first estimation of the parameter settings for MPW. The downside is that neither of the parameters can easily be measured for MPW. If welding windows have to be developed for MPW alternative parameters should be chosen. Previous work [6, 14] mentions the use of impact angle versus standoff, standoff versus charging voltage, or other possible combinations.

\section{WELD QUALITY EVALUATION}

Experiments should be performed to determine the weld quality and thus to evaluate the influence of the process parameters. To test an explosive weld, both non destructive and destructive methods are used. Ultrasonic and radiographic inspection are the most widely used non destructive methods for these welds. For MPW, ultrasonic investigation is not straightforward. Because of the cylindrical configuration of the test specimens, it is difficult to realize a good coupling of the sound waves into the test coupons. An advanced radiographic technique is computerised tomography [15]. Preliminary tests [6] indicate that a source with high power is needed to reach the resolution needed to discover the very small weld flaws (Figure 7a). The cylindrical geometry has the benefit that a leak test can easily performed. 

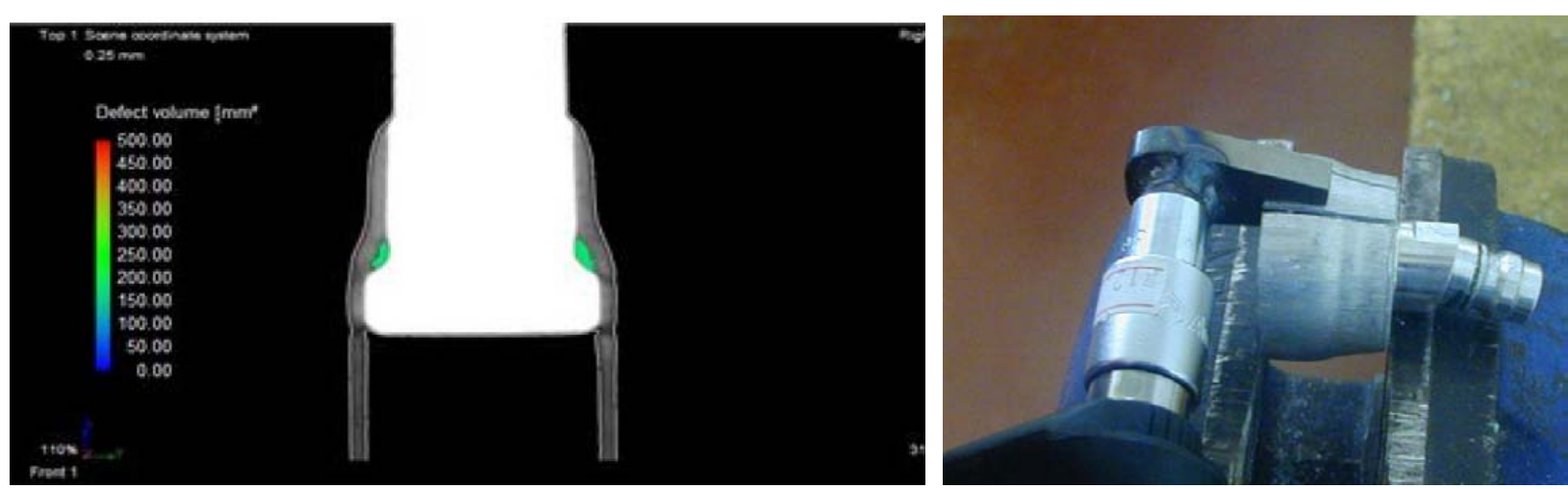

Figure 7 (a) Example of a CT image made of a MP welded specimen [6]

(b) Torque tool being applied on a strip of the weld for a peel test [16]

Because there are not a lot of options for non destructive testing, the majority of tests are of a destructive nature. As can already be concluded from some of the figures, microscopic examination is one of the most important tests which is used for MPW. Next to that fracture tests (tensile, shear, bending), hardness measurements, peeling and chisel tests are an option. Fracture tests are used to evaluate the strength of the weld (i.e. global quality) under load conditions that resemble the in-service loading of the connection. Hardness tests are used to evaluate the presence of hard (and brittle) intermetallic layers (i.e. local quality). A peel test examines the adhesive strength of bonded strips by peeling strips off and recording the required force (Figure $7 \mathrm{~b}$ ). During a chisel test, a chisel is driven into and along the bond interface. The ability of the interface to resist the separating force of the chisel provides an excellent qualitative measure of bond ductility and strength [17]. These tests can also be executed on a circular magnetic pulse welded specimen, but first the flyer tube has to be split in strips [16]. These tests give a characterization of the bonding strength itself. Further tests specifically used for tubular MPW joints are torque tests and tensile or crushing tests [6]. Here the weld strength is compared to the tube strength. If fracture appears in the tube and not in the weld zone, the weld is characterized as sound.

\section{CONCLUSIONS}

Reported knowledge of EXW has shown to be useful in the understanding of the bonding principles and the influence of several process parameters. Both wavelength and amplitude of the wavy joint interface will increase with increasing flyer velocity. The challenge for MPW is the fact that the parameters (impact angle and flyer velocity) are not constant. This results in a changing interface appearance along the length of the weld. The boundaries of the welding windows for explosive welding learn what happens if velocity or impact angle get too high or low. They can however, only give a first estimation of the parameter settings for MPW because the parameters used in those welding windows are hard to measure and adjust in the MPW process. Concerning the quality evaluation of the welds, the used non-destructive and destructive testing methods for EXW have to be adapted to the tubular shape of the products. Tubular products have the benefit of new possibilities such as leak and torque tests. A concluding remark is that a lot of fundamental knowledge on the magnetic pulse welding process still has to be gathered, but the knowledge gained with the EXW process will make this research advance.

\section{ACKNOWLEDGEMENTS}

The first author would like to acknowledge the support of W. De Waele and K. Faes for their help in guiding me into the world of magnetic pulse welding and to provide me with the load of literature necessary to learn more about both processes.

\section{REFERENCES}

1. V. Shribman. Magnetic Pulse welding. PULSAR Ltd. Magnetic Pulse Solutions.

2. figure: www.dynamicmaterials.com

3. Böllinghaus, T., et al., Manufacturing Engineering, in Springer Handbook of Mechanical Engineering, K.-H. Grote and E.K. Antonsson, Editors. 2009, Springer Berlin Heidelberg. p. 523785.

4. Ben-Artzy, A., et al., Wave formation mechanism in magnetic pulse welding. International Journal of Impact Engineering, 2010. 37(4): p. 397-404. 
5. Hokari, H., et al., Magnetic impulse welding of aluminium tube and copper tube with various core materials. Welding International, 1998. 12(8): p. 619 - 626.

6. J. Broeckhove, L.W., Experimental research on magnetic pulse welding of dissimilar metals. 2010, UGent.

7. Durgutlu, A., B. Gulenc, and F. Findik, Examination of copper/stainless steel joints formed by explosive welding. Materials \& Design, 2005. 26(6): p. 497-507.

8. J. B. Perkins, K.L., Z. Akin, Z. Nylund, Explosive Welding of a 12" x 12" x 0.125" ASTM B152 Copper Plate to a 12" x 12" x 0.5" ASTM A516 Gr70 Normalized Steel Plate. 2005, Colorado School of Mines.

9. Mousavi, S.A.A.A. and P.F. Sartangi, Experimental investigation of explosive welding of cptitanium/AISI 304 stainless steel. Materials \& Design, 2009. 30(3): p. 459-468.

10. Durgutlu, A., H. Okuyucu, and B. Gulenc, Investigation of effect of the stand-off distance on interface characteristics of explosively welded copper and stainless steel. Materials \& Design, 2008. 29(7): p. 1480-1484.

11. Acarer, M., B. Gulenc, and F. Findik, The influence of some factors on steel/steel bonding quality on there characteristics of explosive welding joints. Journal of Materials Science, 2004. 39(21): p. 6457-6466.

12. Acarer, M., B. Gulenc, and F. Findik, Investigation of explosive welding parameters and their effects on microhardness and shear strength. Materials \& Design, 2003. 24(8): p. 659-664.

13. Sudarshan, T.S., Explosive Welding of Metals and Its Application - Crossland,B. Journal of Metals, 1983. 35(10): p. 68-68.

14. Loncke, K., An exploratory study into the feasibility of the magnetic pulse forming process. 2009, Ghent Univercity.

15. Computerised Tomography http://www.ndted.org/EducationResources/CommunityCollege/Radiography/AdvancedTechniques/computedtomo graphy.htm.

16. Shribman., V., Magnetic pulse welding of automotive HVAC parts. PULSAR Ltd. Magnetic Pulse Solutions, 2006.

17. welding technology machines. http://www.welding-technology-machines.info/index.htm. 2011. 